\title{
Intratumoral IL-12 and TNF- $\alpha$-Loaded Microspheres Lead To Regression of Breast Cancer and Systemic Antitumor Immunity
}

\author{
Michael S. Sabel, MD, Joseph Skitzki, MD, Lloyd Stoolman, MD, Nejat K. Egilmez, PhD, \\ Edith Mathiowitz, PhD, Nicola Bailey, PhD, Wen-Jian Chang, MD, and Alfred E. Chang, MD
}

\begin{abstract}
Background: Local, sustained delivery of cytokines at a tumor can enhance induction of antitumor immunity and may be a feasible neoadjuvant immunotherapy for breast cancer. We evaluated the ability of intratumoral poly-lactic-acid-encapsulated microspheres (PLAM) containing interleukin 12 (IL-12), tumor necrosis factor $\alpha$ (TNF- $\alpha$ ), and granulocyte-macrophage colony stimulating factor (GM-CSF) in a murine model of breast cancer to generate a specific antitumor response.

Methods: BALB/c mice with established MT-901 tumors underwent resection or treatment with a single intratumoral injection of PLAM containing IL-12, TNF- $\alpha$, or GM-CSF, alone or in combination. Two weeks later, lymph nodes and spleens were harvested, activated with anti-CD3 monoclonal antibodies $(\mathrm{mAb})$ and rhIL-2, and assessed for antitumor reactivity by an interferon $\gamma$ (IFN $\gamma$ ) release assay. Tumor-infiltrating lymphocyte (TIL) analysis was performed on days 2 and 5 after treatment by mechanically processing the tumors to create a single cell suspension, followed by three-color fluorescence-activated cell sorter (FACS) analysis.

Results: Intratumoral injection of cytokine-loaded PLAM significantly suppressed tumor growth, with the combination of IL-12 and TNF- $\alpha$ leading to increased infiltration by polymorphonuclear cells and $\mathrm{CD}^{+} \mathrm{T}$-cells in comparison with controls. The induction of tumor-specific reactive T-cells in the nodes and spleens, as measured by IFN- $\gamma$ production, was highest with IL-12 and TNF- $\alpha$. This treatment resulted in resistance to tumor rechallenge.

Conclusions: A single intratumoral injection of IL-12 and TNF- $\alpha$-loaded PLAM into a breast tumor leads to infiltration by polymorphonuclear cells and $\mathrm{CD}^{+}$T-cells with subsequent tumor regression. In addition, this local therapy induces specific antitumor T-cells in the lymph nodes and spleens, resulting in memory immune response.
\end{abstract}

Key Words: Breast cancer-IL-12-Immunotherapy-Microspheres-TNF- $\alpha$.

Although adjuvant chemotherapy and hormonal therapy have improved the overall survival of breast cancer patients after surgery, the persistence of tumor cells remains a major cause of recurrence and death. ${ }^{1}$ Many of

\footnotetext{
Received March 7, 2003; accepted October 16, 2003.

From the Division of Surgical Oncology (MSS, JS, W-JC, AEC) and Department of Pathology (LS), University of Michigan, Ann Arbor, Michigan; SUNY at Buffalo (NKE), Buffalo, New York; and Brown University (EM, NB), Providence, Rhode Island.

Presented at the 2003 Society of Surgical Oncology Cancer Symposium, Los Angeles, California, March, 5-9, 2003.

Address correspondence and reprint requests to: Michael S. Sabel, MD, Asst. Professor of Surgery, Division of Surgical Oncology, University of Michigan Comprehensive Cancer Center, 3304 Cancer Center, 1500 East Medical Center Drive, Ann Arbor, MI 48109-0932; Fax: 734-647-9647; E-mail: msabel@umich.edu.

Published by Lippincott Williams \& Wilkins (C) 2004 The Society of Surgical Oncology, Inc.
}

these disseminated tumor cells rest in the G0 phase of the cell cycle. ${ }^{1-3}$ Because these nonproliferating cells are often resistant to chemotherapeutic agents, ${ }^{2,3}$ many investigators have looked toward immunotherapy in the adjuvant setting as an alternative approach to reducing recurrence rates.

Breast cancer presents several unique challenges to the use of immunotherapy. Only a few tumor antigens have been identified in breast cancer, and many of these are expressed in only a relatively small fraction (20\% to $30 \%$ ) of breast tumors, ${ }^{4}$ hampering the success of peptide vaccines or allogeneic cellular vaccines. Autologous tumor cell vaccines have been investigated but have demonstrated disappointing clinical responses. ${ }^{5}$ More promising results have been demonstrated with the use of genetically modified tumor cell vaccines, but this re- 
quires the creation of cell lines for ex vivo manipulation. This has been extremely difficult with breast cancer, where less tumor is available for procurement and the establishment of cell lines has proved challenging. In addition, ex vivo genetic manipulation can be associated with limited transfection efficiency and is costly and time-consuming. All these factors limit its applicability to the clinical setting. The adenoviral delivery of genes encoding cytokines directly to the tumor in situ may circumvent some of these problems but carries several safety concerns.

We have previously described our experience with the intratumoral injection of cytokine-encapsulated biodegradable polymer microspheres, demonstrating that the local and sustained release of interleukin 12 (IL-12) in the tumor microenvironment results in long-term, tumorspecific immunity. ${ }^{6}$ Utilized in the neoadjuvant setting, intratumoral injection of IL-12-loaded microspheres into the tumor prior to surgical resection was able to decrease both local and distant tumor recurrence. ${ }^{7}$ Given the limitations of many immunotherapeutic approaches and the ease of direct intratumoral injections, the use of cytokine-loaded microspheres seems well-suited to the treatment of breast cancer. To investigate this, we evaluated the effect of intratumoral injections of cytokine-loaded microspheres on the subcutaneous growth of the murine MT-901 breast tumor and the stimulation of both a regional and systemic tumor-specific immune response. We report here that the intratumoral delivery of various cytokine-loaded poly-lactic-acid microspheres (PLAM) can eradicate established tumors and result in significantly enhanced T-cell reactivity of tumor-draining lymph node (TDLN) cells and splenocytes. PLAM loaded with the combination of IL- 12 and TNF- $\alpha$ yielded greater local and systemic response than either alone.

\section{MATERIALS AND METHODS}

\section{Animals}

Six- to 8-week-old female BALB/c mice were purchased from The Jackson Laboratory (Bar Harbor, NE) and maintained in specific pathogen-free conditions at the Animal Maintenance Facility of the University of Michigan Medical Center.

\section{Tumors}

MT-901 is a subline of a murine tumor cell line derived from a dimethylbezanthracene-induced mammary carcinoma in the BALB/c strain. ${ }^{8}, 9$ This tumor is weakly immunogenic and expresses MHC class I but not MHC class II molecules. RENCA is an immunogenic murine renal cell carcinoma of spontaneous origin in the
BALB/c strain..$^{10}$ Line-1 is a BALB/c lung alveolar carcinoma cell line. ${ }^{11}$ Cell lines were maintained in complete media consisting of RPMI 1640 supplemented with $10 \%$ heat-activated fetal bovine serum, $.1 \mathrm{mM}$ nonessential amino acids, $1 \mu \mathrm{M}$ sodium pyruvate, $100 \mu \mathrm{g} / \mathrm{mL}$ streptomycin, 100 units $/ \mathrm{mL}$ penicillin, $50 \mu \mathrm{g} / \mathrm{mL}$ gentamycin, and $.5 \mu \mathrm{g} / \mathrm{mL}$ fungizone, all from Life Technologies (Grand Island, NY). In vivo generation of tumors was accomplished by subcutaneous injection of BALB/c mice with $3 \times 10^{6}$ viable MT-901 cells. Tumors were measured every other day in two perpendicular dimensions $(\mathrm{a}=$ length, $\mathrm{b}=$ width) with a Vernier caliper, and the size was recorded as volume $\left(\mathrm{mm}^{3}\right.$, as calculated by $\left.a^{*} b^{2} / 2\right)$. Statistical significance between groups $(n=5)$ was calculated with an unpaired Student's $t$-test, with a $P$ value $<.05$ considered statistically significant.

\section{Microspheres}

A phase-inversion nanoencapsulation technique was used for encapsulation of cytokines, as described previously. ${ }^{12}$ In brief, bovine serum albumin (BSA, RIA grade; Sigma Chemical Co., St. Louis, MO), PLA $\left\{M_{\mathrm{r}}\right.$ 24,000 and $M_{\mathrm{r}} 2,000$ [1:1, w/w], Birmingham Polymers, Birmingham, AL $\}$, and recombinant cytokine in methylene chloride (Fisher, Pittsburgh, PA) were rapidly poured into petroleum ether (Fisher) for formation of microspheres. Microspheres were filtered and lyophilized overnight for complete removal of solvent. Four formulations containing $1 \% \mathrm{BSA}(\mathrm{w} / \mathrm{w})$ were produced: (1) control (no cytokines); (2) murine IL-12 \{ $\sim 5 \mathrm{~g} / \mathrm{mg}$ of PLA; RandD Systems, Minneapolis, MN); (3) murine granulocyte-macrophage colony stimulating factor (GMCSF: $\sim 5 \mu \mathrm{g} / \mathrm{mg}$ of PLA; Biosource International, Camarillo, CA); and (4) murine tumor necrosis factor $\alpha$ (TNF- $\alpha$ : $\sim 5 \mu \mathrm{g} / \mathrm{mg}$ of PLA; RandD Systems). Scanning electron micrographs previously demonstrated that these microspheres are $1-5 \mu \mathrm{m}$ in diameter and are easily injectable with a 27 -gauge needle.

\section{Tumor-Draining LN Cells and Activation}

At varying time points after inoculation, tumor-draining LN cells (TDLN) were removed aseptically. Lymphoid cell suspensions were prepared by mechanical dissociation with 25 -gauge needles and pressed with the blunt end of a 10-mL plastic syringe in RPMI 1640. The cells were activated with $1 \mu \mathrm{g} / \mathrm{mL}$ anti-CD3 monoclonal antibody $(\mathrm{mAb})$ immobilized in 24 -well plates $\left(4 \times 10^{6}\right.$ cells $/ 2 \mathrm{~mL} /$ well) for 2 days. ${ }^{13}$ The $\mathrm{LN}$ cells were subsequently cultured in recombinant human IL-2 (Chiron Therapeutics, Emeryville, CA) at $60 \mathrm{IU} / \mathrm{mL}$ IL-2 for 3 days at $2 \times 10^{5}$ cells $/ \mathrm{mL}$. 


\section{Splenocytes and Activation}

Spleen cells obtained from the BALB/c mice were treated with ammonium chloride-potassium lysis buffer (.83\% ammonium chloride, .1\% $\mathrm{KHCO} 3$, and $.004 \%$ EDTA) for 1 minute to deplete erythrocytes and were washed twice with HBSS. Splenocytes were then activated with $1 \mu \mathrm{g} / \mathrm{mL}$ anti-CD3 $\mathrm{mAb}$ immobilized in 24well plates $\left(4 \times 10^{6}\right.$ cells $/ 2 \mathrm{~mL} /$ well $)$ for 2 days. ${ }^{13}$ The LN cells were subsequently cultured in $60 \mathrm{IU} / \mathrm{mL}$ IL-2 for 3 days at $2 \times 10^{5}$ cells $/ \mathrm{mL}$.

\section{Measurement of In Vitro Cytokine Release}

Activated TDLN or splenocytes $\left(1 \times 10^{6}\right)$ were cocultured with $5 \times 10^{5}$ MT-901 tumor cells irradiated to $15,000 \mathrm{cGy}$ in $2 \mathrm{~mL}$ of complete media (CM) per well of a 24-well tissue culture plate. IL-2 (4 IU/mL) was added at the beginning of the culturing for 48 hours at $37^{\circ} \mathrm{C}$. the supernatants were collected and, after centrifugation, analyzed for interferon- $\gamma($ IFN $\gamma)$ with commercially available ELISAs from PharMingen (San Diego, CA). A standard curve starting at $1000 \mathrm{U} / \mathrm{mL}$ was established, with 11 serial twofold dilutions performed. Experimental values were computed with the use of regression analysis.

\section{Tissue Processing for Tumor-Infiltrating Lymphocyte (TIL) Analysis}

MT 901 tumors were harvested on days 2 and 5 after microsphere injection. The tumor and surrounding tissue (within $2 \mathrm{~mm}$ of tumor) were removed, minced with scissors to $\sim 1-\mathrm{mm}^{3}$ pieces, and processed mechanically with a Medimachine (DakoUSA, Carpinteria, CA). Processing involved placing the tissue in a microbladeequipped polyethylene chamber (Medicon; CONSULT.S., Torino, Italy) $35 \mu \mathrm{M}$ in size, adding $.5 \mathrm{~mL}$ of PBS, and cycling the sample in the Medimachine for 45 seconds. The contents of the Medicon were collected via a 1-cc syringe and passed through a $50-\mu \mathrm{M}$ filter to create a single cell suspension. The processing was performed twice for each sample.

\section{Fluorescence-Activated Cell Sorter (FACS) Analysis}

Processed tumor was enumerated for cell counts and then stained for three-color FACS analysis with the fluorescein isothiocyanate(FITC)-conjugated $\mathrm{mAb}$ anti$\mathrm{CD}$, phycoerythrin(PE)-conjugated $\mathrm{mAb}$ anti-CD4, PEconjugated mAb NK1.1, PE-conjugated mAb GR-1, PEconjugated mAb DX5, and CyChrome(CYC)-conjugated
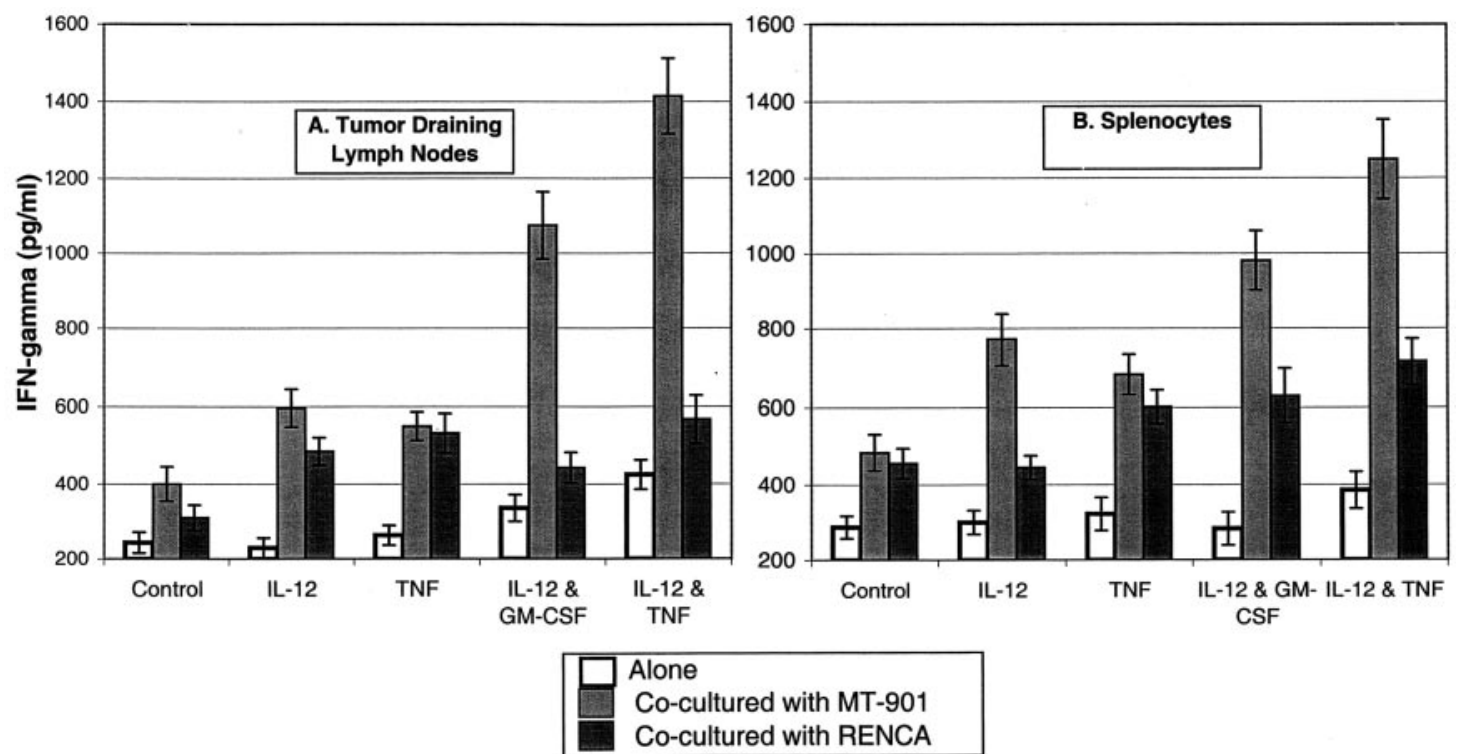

FIG. 1. Inoculation of MT-901 with cytokine-loaded PLAM induces tumor-specific pre-effector cells both regionally and systemically. BALB/c mice (5 mice per group) were co-inoculated with $3 \times 10^{6}$ viable MT-901 cells and poly-lactic-acid microspheres encapsulating IL-12, TNF- $\alpha$, and GM-CSF alone or in combination. Control mice were co-inoculated with BSA-loaded microspheres. Nine days later, TDLN and spleens were removed aseptically. Lymphoid single-cell suspensions were activated with anti-CD3 mAb for 2 days and then cultured in $60 \mathrm{IU} / \mathrm{mL}$ IL-2 for 3 days. Activated TDLN or splenocytes were cocultured with irradiated MT-901 or RENCA tumor cells. The supernatants were collected and analyzed for IFN $\gamma$ production. Control or naïve lymphoid cells also did not release cytokines. (A) TDLN from mice inoculated with MT-901 and PLAM loaded with the combination of IL-12 and GM-CSF or IL-12 and TNF- $\alpha$ revealed the tumor-specific release of IFN $\gamma$ in response to MT-901 tumor cells and not to Renca cells. (B) Splenocytes from mice inoculated with MT-901 and PLAM loaded with the combination of IL-12 and GM-CSF or IL-12 and TNF- $\alpha$ also showed a dramatic increase in the release of IFN $\gamma$ in response to MT-901 tumor cells but not Renca cells. In both the TDLN and spleens, the highest tumor-specific response was seen with PLAM loaded with the combination of IL-12 and TNF- $\alpha$. Error bars represent standard deviation (SD). 
mAb anti-CD45 (all obtained from PharMingen). Isotype controls for each of the antibodies were used to define specific binding. All samples were fixed in a $2 \%$ buffered formalin solution and read 24 hours after labeling. Prior to analysis on a Coulter XL (Beckman Coulter, Chaska, MN), a known quantity of $15 \mu \mathrm{m}$ polystyrene microbeads (Bangs Laboratories, Fishers, IN) were added to each sample to calculate the absolute number of cells of interest during analysis, with use of the following formula: $\{$ number of events of interest $\} *$ number of beads added to sample\} / \{number of beads sampled\}. Analysis of tumor tissue involved the collection of more than $1,500,000$ events and use of the WinList 5.0 program (Verity Software House, Topsham, ME). CD4, $\mathrm{CD} 8$, polymorphonuclear $(\mathrm{PMN})$, and natural killer (NK) cell events were gated only on CD45-positive events. Absolute cell counts were expressed as a function of individual tumor size.

\section{Statistical Analysis}

Statistical significance between groups was calculated with an unpaired Student's $t$-test, with a $P$ value $<.05$ considered statistically significant.

\section{RESULTS}

Inoculation of MT-901 with IL-12 and GM-CSF or TNF- $\alpha$-loaded PLAM induces tumor-specific pre-effector cells both regionally and systemically. To identify the cytokines (alone or in combination) that could stimulate a regional or systemic response, mice were inoculated with MT-901 tumor cells in the flank in combination with $1 \mathrm{mg}$ of cytokine-encapsulated microspheres (for combination therapy, $.5 \mathrm{mg}$ of each cytokine was used; for control groups, BSA-loaded microspheres were used). Treatment groups comprised five mice each. Nine days later the mice were killed and had TDLN and spleens harvested for anti-CD3/IL-2 activation. TDLN from mice inoculated with MT-901 alone demonstrated no release of IFN $\gamma$ in response to tumor stimulation. In this assay, control or naïve lymphoid cells also did not release cytokines. TDLN from mice inoculated with MT901 and either IL-12-loaded PLAM or TNF- $\alpha$-loaded PLAM showed an increased release of IFN $\gamma$ in response to tumor stimulation, in comparison with nonstimulated lymphocytes. This response, however, was nonspecific, as it was stimulated by both MT-901 and Renca cells. TDLN from mice inoculated with MT-901 and PLAM loaded with the combination of IL-12 and GM-CSF or the combination of IL- 12 and TNF- $\alpha$ revealed the tumorspecific release of IFN $\gamma$ only in response to MT-901 tumor cells and not to Renca cells (Fig. 1A).
Splenocytes from mice inoculated with MT-901 alone also demonstrated minimal release of IFN $\gamma$ in response to tumor stimulation. Splenocytes from mice inoculated with MT-901 and IL-12-loaded PLAM showed a mild tumor-specific increase in IFN $\gamma$, while those inoculated with TNF- $\alpha$ did not. Again, however, splenocytes from mice inoculated with MT-901 and PLAM loaded with the combination of IL-12 and GM-CSF or IL-12 and TNF- $\alpha$ resulted in a dramatic increase in the release of IFN $\gamma$ in response to MT-901 tumor cells but not Renca cells (Fig. 1b). In both the TDLN and spleens, the highest tumor-specific response was seen with PLAM loaded with the combination of IL-12 and TNF- $\alpha$.

\section{Treatment of Established Tumors with an Intratumoral Injection of IL-12 and GM-CSF or TNF- $\alpha$-Loaded PLAM Causes Tumor Regression}

On the basis of these results, we next sought to investigate whether this approach would be effective in the treatment of established tumors. Balb/c mice were inoculated subcutaneously with MT-901 cells in the right flank. Treatment groups comprised five mice each. Eleven days after inoculation, when all mice had palpable subcutaneous tumors, mice were treated by intratumoral injection of cytokine-loaded microspheres. Control mice were injected with a single injection of BSAloaded microspheres at a dose of $2 \mathrm{mg}$ PLA/tumor. Mice

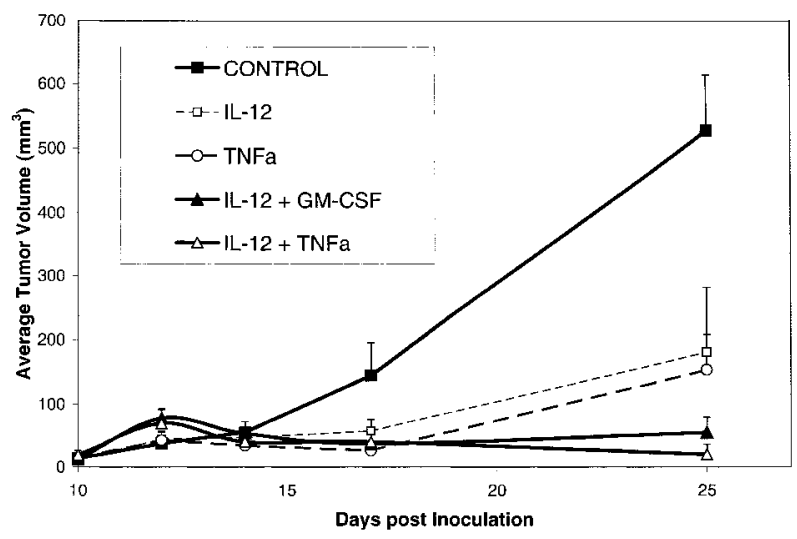

FIG. 2. Effect of intratumoral injection of cytokine-loaded PLAM on primary tumor growth. Balb/c mice (five to 10 mice per group) were inoculated subcutaneously with $3 \times 10^{6}$ MT-901 cells in the right flank. Eleven days later, palpable tumors were treated by intratumoral injection of cytokine-loaded microspheres (or BSA-loaded microspheres in control mice). Tumors were measured every other day in two perpendicular dimensions $(\mathrm{a}=$ length, $\mathrm{b}=$ width) and the size was recorded as a volume $\left(\mathrm{mm}^{3}\right)$, as calculated by $\mathrm{a}^{*} \mathrm{~b}^{2} / 2$. Tumor suppression was seen with intratumoral injection of IL-12 alone and TNF- $\alpha$ alone. Tumor regression was seen with the intratumoral injection of IL-12 in combination with GM-CSF and TNF- $\alpha$. The addition of TNF- $\alpha$ to IL-12 significantly improved the results in comparison with IL-12 alone $(P=.03)$. Error bars represent the standard deviation (SD). 


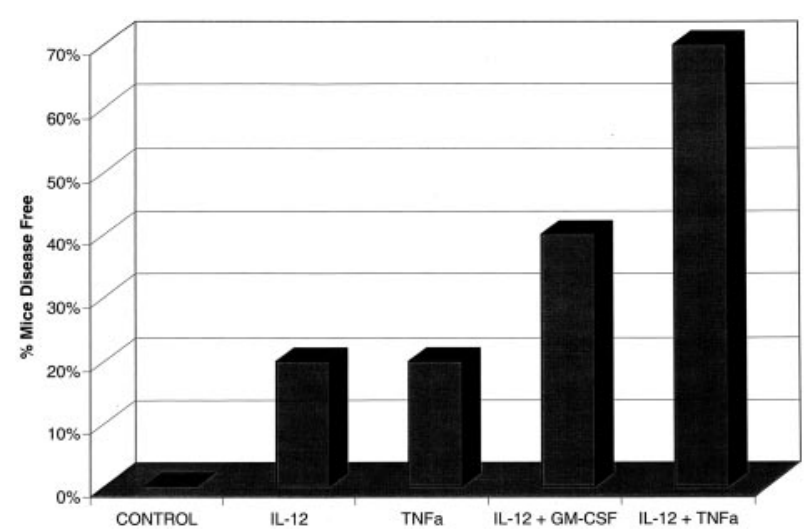

FIG. 3. Percentage of mice rendered tumor-free after intratumoral injection of cytokine-loaded poly-lactic-acid microspheres.

treated with IL-12 or TNF- $\alpha$-loaded microspheres were also treated at a dose of $2 \mathrm{mg}$ PLA/tumor. Mice treated with the combination of IL-12 and GM-CSF or IL-12 and TNF- $\alpha$ were treated by two injections of each cytokine at a dose of $1 \mathrm{mg}$ PLA/tumor. By day 25 after inoculation, tumor size in the untreated group averaged $526 \mathrm{~mm}^{3}$ (Fig. 2). Tumor suppression was seen with both intratumoral injection of IL-12 (average tumor size, 181 $\mathrm{mm}^{3} ; P=.04$ ) and TNF- $\alpha$ (average tumor size, 153 $\mathrm{mm}^{3} ; P=.03$ ). Tumor regression was seen with the intratumoral injection of IL-12 in combination with GM-
CSF (average tumor size, $55 \mathrm{~mm}^{3} ; P=.004$ in comparison with controls) and TNF- $\alpha$ (average tumor size, 9 $\mathrm{mm}^{3} ; P<.0001$ in comparison with controls). The addition of TNF- $\alpha$ to IL-12 significantly improved the results in comparison with IL-12 alone $(P=.03)$, and this combination of IL-12 and TNF- $\alpha$ resulted in the highest percentage of tumor-free mice (Fig. 3).

\section{Treatment of Established Tumors with an} Intratumoral Injection of IL-12 and TNF- $\alpha$-Loaded PLAM Induces Tumor-Specific Pre-effector Cells both Regionally and Systemically

Mice with MT-901 tumors treated by intratumoral injection of cytokine-loaded PLAM were killed 14 days later, and both TDLN and spleens were harvested for anti-CD3/IL-2 activation. TDLN from mice treated with BSA-loaded PLAM demonstrated no release of IFN $\gamma$ in response to tumor stimulation. TDLN from mice treated with either PLAM loaded with the combination of IL-12 and GM-CSF or IL-12 and TNF- $\alpha$ showed a dramatically higher release of IFN $\gamma$ in response to MT-901 tumor cells than to Line-1 cells (Fig. 4a).

Splenocytes from mice treated with IL-12 or TNF- $\alpha$ alone again demonstrated minimal release of IFN $\gamma$ in response to tumor stimulation. The combination of IL-12 and GM-CSF, which has been effective in previous assays, failed to demonstrate a significant systemic re-

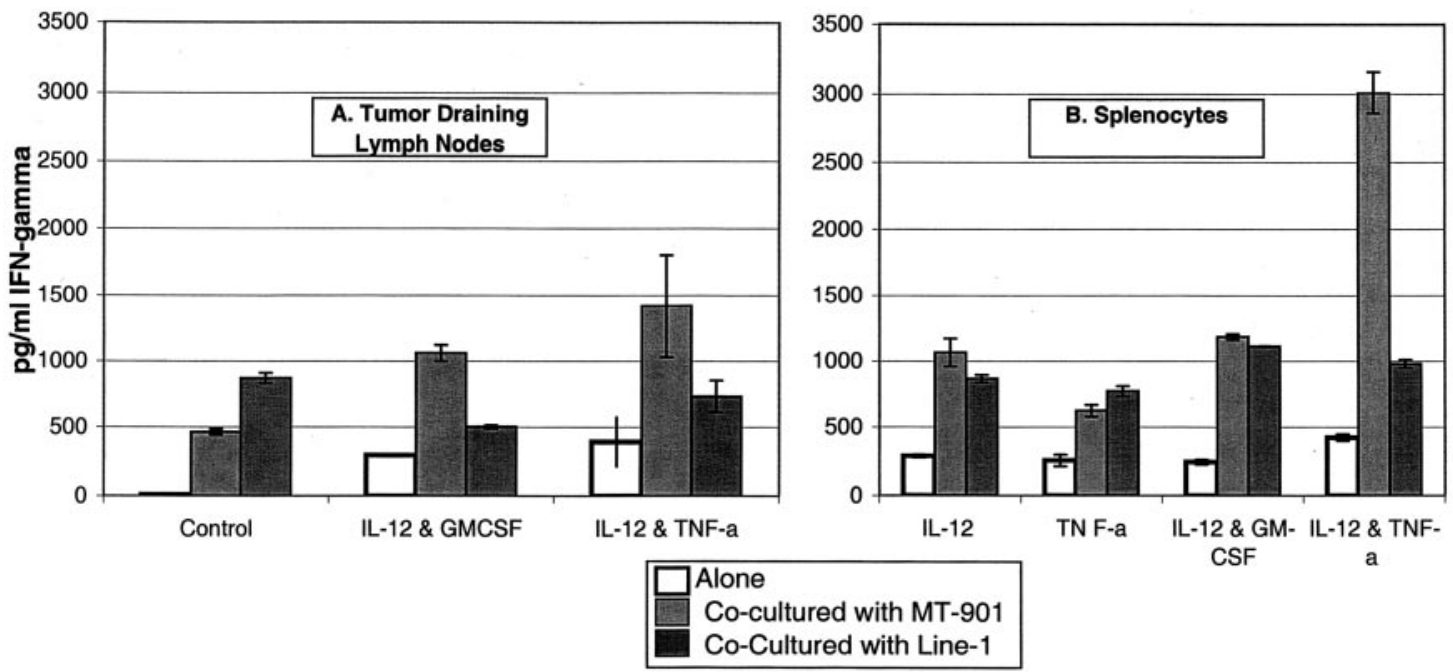

FIG. 4. Treatment of MT-901 tumors with IL-12 and TNF- $\alpha$-loaded PLAM induces systemic tumor specific pre-effector cells. Balb/c mice (five mice per group) were inoculated subcutaneously with $3 \times 10^{6}$ MT-901 cells in the right flank. Eleven days later, palpable tumors were treated by intratumoral injection of cytokine-loaded microspheres (or BSA-loaded microspheres in control mice). TDLN and spleens were harvested and single-cell suspensions were activated with anti-CD3 mAb for 2 days and then cultured in $60 \mathrm{IU} / \mathrm{mL}$ IL-2 for 3 days. Activated TDLN or splenocytes were cocultured with irradiated MT-901 or Line-1 tumor cells and then analyzed for IFN $\gamma$ production. (A) TDLN from mice treated with PLAM loaded with the combination of IL-12 and GM-CSF or IL-12 and TNF- $\alpha$ resulted in a substantial tumor-specific release of IFN $\gamma$ in response to MT-901 tumor cells. The splenocytes of mice treated by PLAM loaded with the combination of IL-12 and GM-CSF failed to demonstrate a significant antitumor response in splenocytes. (B) Splenocytes from mice treated with IL-12 and TNF- $\alpha$-loaded PLAM showed a significant tumor-specific release of IFN $\gamma$ in response to MT-901 tumor cells. Error bars represent standard deviation (SD). 
sponse in the treatment of an established tumor, as measured by tumor-specific IFN $\gamma$ release in several experiments. Splenocytes from mice treated with PLAM loaded with the combination of IL-12 and TNF- $\alpha$, however, showed a significant tumor-specific release of IFN $\gamma$ in response to MT-901 tumor cells (Fig. 4b).

\section{Intratumoral Injection of Cytokine-Loaded PLAM} Resulted in the Infiltration of PMN Cells and $\mathrm{CD8}^{+}$ Cytotoxic T-Cells at the Site of the Tumor

Growth curves after intratumoral injection of cytokine-loaded PLAM suggested that IL-12 or TNF- $\alpha$ alone led to tumor suppression, whereas the combination of IL-12 and TNF- $\alpha$ led to tumor regression (Fig. 2), with eradication of tumor in $70 \%$ of treated mice (Fig. 3).
Tumor regression was seen approximately 3 days after treatment. To further identify the cellular component of the immune response responsible for tumor regression, three-color FACS analysis was used to enumerate the tumor-infiltrating lymphocytes at days 2 and 5 after treatment with microspheres encapsulating IL-12, TNF- $\alpha$, or the combination (Fig. 5). Control mice were treated with BSA-loaded microspheres. The results are shown in Fig. 6. Treatment with control microspheres resulted in minimal amounts of infiltrating lymphocytes on both day 2 and day 5. Treatment with IL-12 in combination with TNF- $\alpha$ resulted in a statistically significant rise in PMN cells within the tumor microenvironment $(P=.04)$. Despite the fact that the NK-stimulating properties of IL-12 have been well described, NK

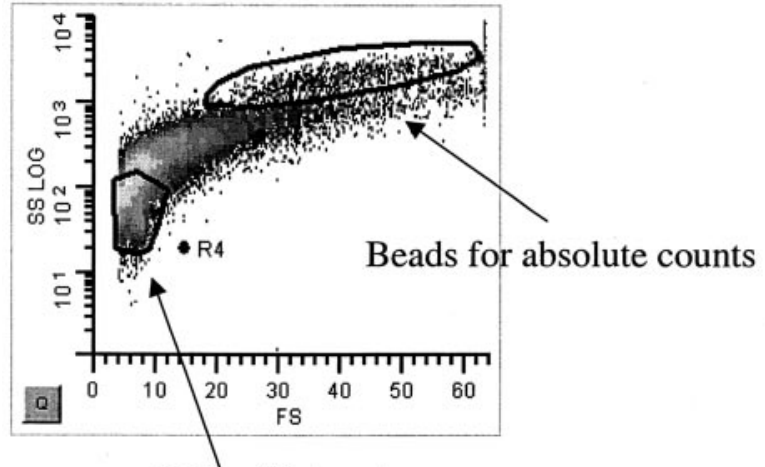

Cells of interest

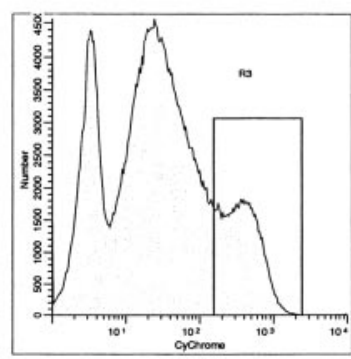

CD45 cyc

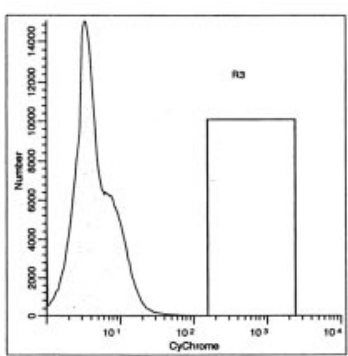

isotype control
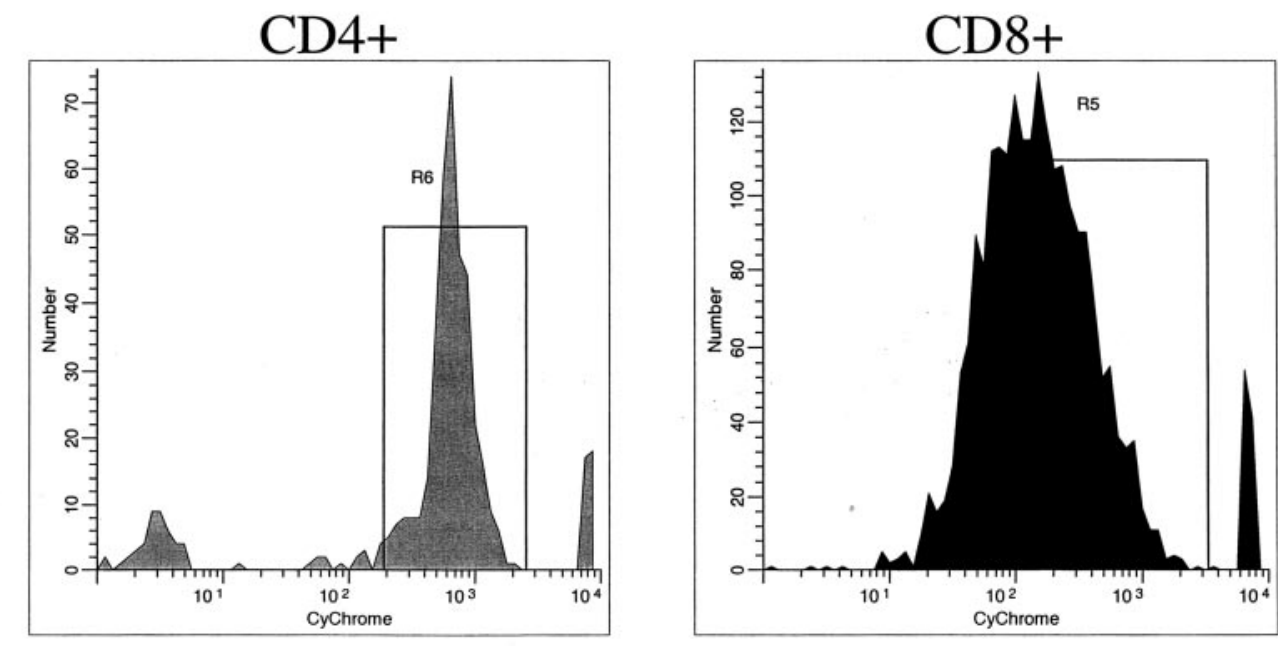

FIG. 5. FACS analysis of tumor-infiltrating lymphocytes. Treated MT 901 tumors were removed, processed mechanically, and passed through a $50-\mu \mathrm{M}$ filter to create a single-cell suspension. Cells were then enumerated for cell counts and then stained for three-color FACS analysis with FITC-conjugated mAb anti-CD8, PE-conjugated mAb anti-CD4, and CYC-conjugated mAB anti-CD45. Isotype controls for each of the antibodies were used to define specific binding. All samples were fixed in a $2 \%$ buffered formalin solution and read 24 hours after labeling. Prior to analysis, a known quantity of $15 \mu \mathrm{m}$ polystyrene microbeads was added to each sample to calculate the absolute number of cells of interest during analysis. CD4 and CD8 cells were stringently gated on the high CD45 ${ }^{+}$population to eliminate background signal from specific binding. 


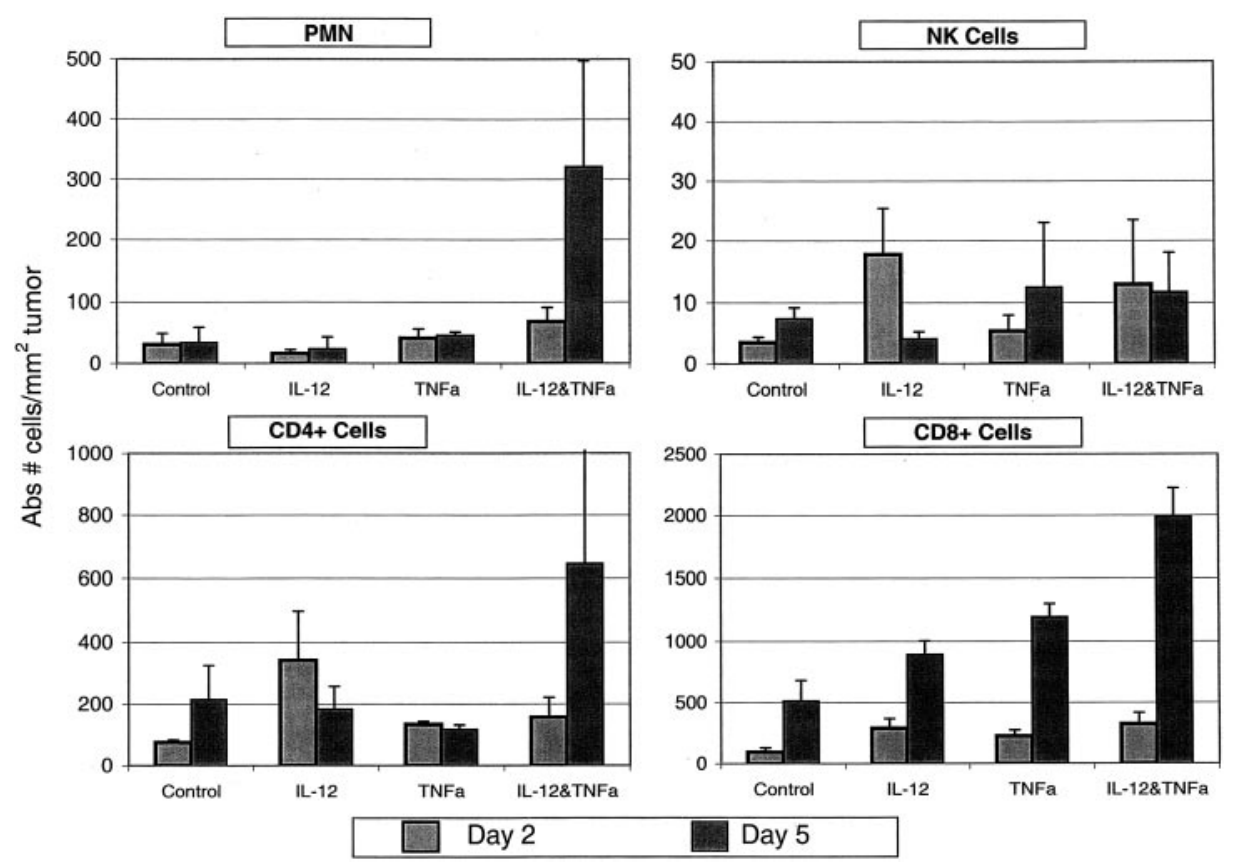

FIG. 6. Intratumoral injection of cytokine-loaded PLAM resulted in the infiltration of PMN cells and CD8 $8^{+}$cytotoxic T-cells at the site of the tumor. $\mathrm{Balb} / \mathrm{c}$ mice (five mice per group) were inoculated subcutaneously with $3 \times 10^{6}$ MT-901 cells in the right flank. Twelve days later, palpable tumors were treated by intratumoral injection of cytokine-loaded microspheres (or BSA-loaded microspheres in control mice). Mice were killed on days 2 and 5 after treatment. Treatment with IL-12 in combination with TNF- $\alpha$ resulted in a statistically significant rise in PMN cells within the tumor microenvironment $(P=.04)$. There was no change in NK cells within the treated tumors for any of the groups. IL-12 or TNF-a alone had no effect on $\mathrm{CD} 4^{+} \mathrm{T}$-helper cells. The combination appeared to lead to an increase in $\mathrm{CD} 4^{+}$infiltration, but this was not statistically significant $(P=.18)$ Both IL-12 and TNF- $\alpha$ alone led to significant increases in tumor infiltration by CD8 ${ }^{+}$cytotoxic T-cells $(P=.02$ and $P=.004$, respectively). The intratumoral injection of PLAM loaded with the combination of IL-12 and TNF- $\alpha$ led to a statistically significant increase in CD $8^{+}$cells in comparison with control groups $(P=.001)$ or either cytokine alone $(P=.002$ for IL-12 alone and $P=.006$ for TNF- $\alpha$ alone $)$.

cells did not appear to be responsible for tumor regression, as there was no change in NK cells within the treated tumors for any of the groups. IL- 12 or TNF- $\alpha$ alone had no effect on $\mathrm{CD}^{+}{ }^{+}$T-helper cells. The combination appeared to lead to an increase in $\mathrm{CD}^{+}{ }^{+}$infiltration, but this was not statistically significant $(P=.18)$. However, both IL-12 and TNF- $\alpha$ alone led to significant increases in tumor infiltration by $\mathrm{CD} 8^{+}$cytotoxic T-cells $(P=.02$ and $P=.004$, respectively). The intratumoral injection of PLAM loaded with the combination of IL-12 and TNF- $\alpha$ led to a statistically significant increase in $\mathrm{CD} 8^{+}$cells in comparison with the control groups $(P=$ $.001)$ or either cytokine-alone group $(P=.002$ for IL-12 alone and $P=.006$ for TNF- $\alpha$ alone).

\section{Intratumoral Injection of IL-12 and TNF- $\alpha$-Loaded PLAM Resulted in Immune Memory}

In a separate experiment, Balb/c mice were inoculated with a subcutaneous injection of $3 \times 10^{6}$ MT-901 cells in the right flank. On day 11, when all mice had palpable subcutaneous tumors, mice underwent either surgical excision of the tumor or injection with $1 \mathrm{mg}$ PLA/tumor of IL-12 and either TNF- $\alpha$ ( $1 \mathrm{mg}$ PLA/tumor) or GMCSF (1 mg PLA/tumor) (10 mice per group). Three weeks later, all tumor-free mice were re-inoculated on the opposite flank with an identical tumorigenic dose of MT-901 cells. By day 10 after rechallenge, $80 \%$ of mice treated by surgical excision had tumors, whereas none of the mice treated by intratumoral IL- 12 and TNF- $\alpha$ PLAM had evidence of tumor growth (Fig. 7) $(P<.002$ by $\chi^{2}$ test).

\section{DISCUSSION}

Slow-release polymer microspheres can be used to achieve the paracrine delivery of cytokines to the tumor microenvironment after a single intratumoral injection. This approach has the potential to generate an effective systemic response with minimal side effects. ${ }^{14}$ The results described here demonstrate that a single injection of IL-12 and TNF- $\alpha$ encapsulated microspheres into a primary breast tumor initiates infiltration with PMN cells and $\mathrm{CD}^{+}$cytotoxic T-cells, leading to regression of the tumor. In addition, this process can induce tumor-spe- 


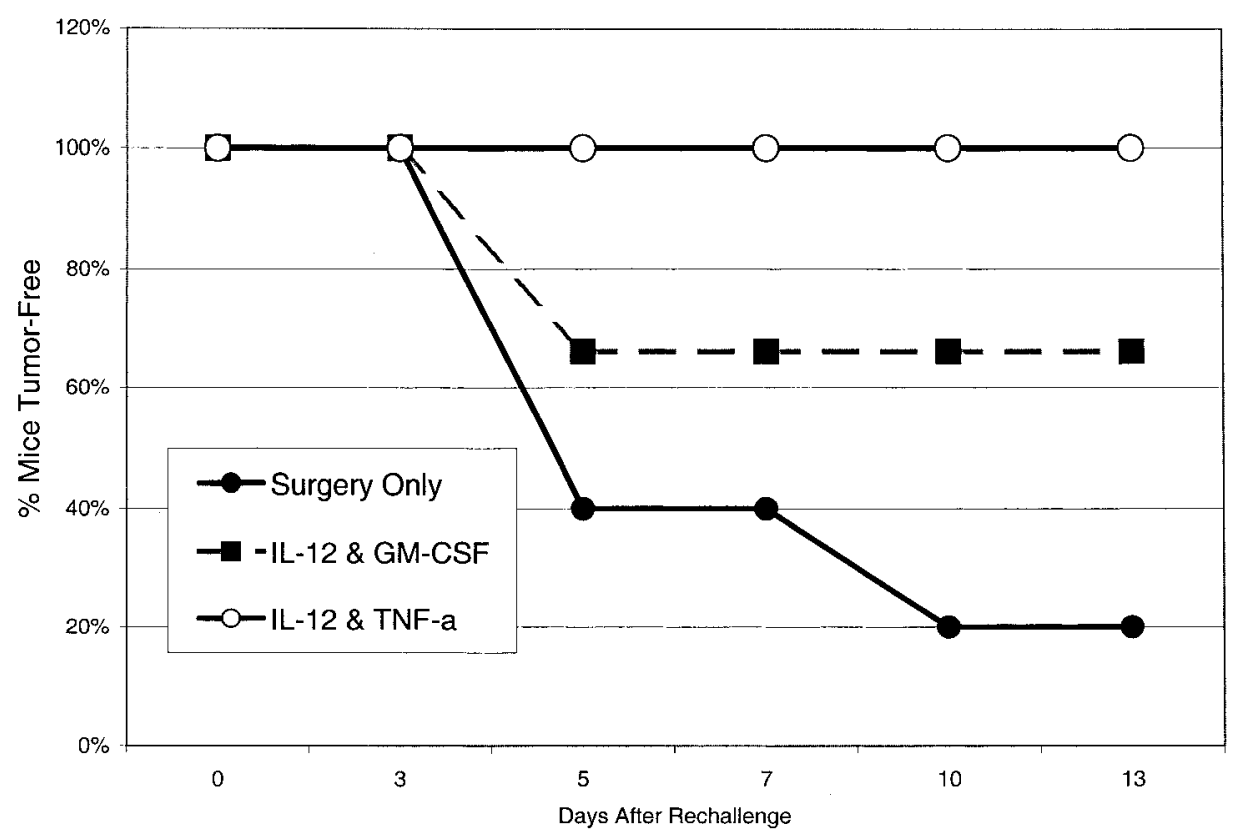

FIG. 7. Mice cured by intratumoral injection of IL-12 and TNF- $\alpha$-loaded PLAM develop long-term immunity in comparison with the results of surgical excision alone. Balb/c mice (10 mice per group) were inoculated with a subcutaneous injection of $3 \times 10^{6}$ MT-901 cells in the right flank On day 12, when all mice had palpable subcutaneous tumors, mice underwent either surgical excision of the tumor or injection with PLAM loaded with IL-12 and TNF- $\alpha$ or GM-CSF. Three weeks later, all successfully treated mice were then re-inoculated on the opposite flank with an identical tumorigenic dose of MT-901 cells. By day 10 after rechallenge, $80 \%$ of mice treated by surgical excision had tumors but none of the mice treated by intratumoral IL-12 and TNF- $\alpha$ PLAM had evident tumor growth $\left(P<.002\right.$ by $\left.\chi^{2}\right)$.

cific T-cells both regionally-in the tumor-draining lymph nodes-and systemically in the spleen. This immune response results in a memory response, as evidenced by resistance to tumor rechallenge.

We have previously described an in situ cytokine delivery system utilizing slow-release polymer microspheres. This achieves a sustained release of cytokines into the tumor microenvironment, resulting in a local inflammatory response. We hypothesize that the necrosis of the primary tumor provides large doses of tumor antigen locally and the subsequent induction of an adaptive tumor-specific T-cell response. ${ }^{6,15}$ In our initial experiments IL-12 demonstrated the most promise for single cytokine therapy with this approach ${ }^{6}$ and has shown tremendous potential in multiple models. ${ }^{16-19} \mathrm{IL}-12$ is a potent proinflammatory cytokine, with the ability to induce rapid activation of innate and anti-angiogenic mechanisms ${ }^{21,22}$ and to promote the development of a Th1-type cellular response. ${ }^{23}$ In this series of experiments, intratumoral injection of IL-12-loaded PLAM did demonstrate some benefit, causing a significant tumor infiltration of $\mathrm{CD}^{+}$cells and tumor regression in comparison with controls, but it did not result in an effective systemic antitumor response, particularly when used in the treatment of established tumors.
Several groups using different models of cytokinebased immunotherapies have demonstrated that the combination of IL-12 with other pro-inflammatory cytokines can improve this response. In a previous report, we demonstrated that the combination of IL-12 and GMCSF was significantly better than IL-12 alone in our model, resulting in adaptive antitumor responses that could eradicate disseminated disease. ${ }^{15}$ Other researchers have also demonstrated the benefits of combining IL-12 with GM-CSF, ${ }^{24,25}$ as well as with TNF- $\alpha .^{25-28}$ This study confirms that in a murine model of breast cancer, PLAM loaded with the combination of IL-12 and GMCSF was effective and significantly better than IL-12 alone. Co-inoculation with MT-901 cells led to a regional and systemic tumor-specific response and also caused tumor regression when injected intratumorally into established tumors. However, the most effective combination was IL-12 and TNF- $\alpha$, surpassing IL-12 and GM-CSF in both tumor regression and the percentage of mice cured, as well as the regional and systemic response. In addition, this combination was shown to result in immune memory capable of preventing tumor growth at a subsequent rechallenge. The success of these combinations is not surprising, given that TNF- $\alpha$ synergizes with IL-12 for IFN- $\gamma$ production, skewing a CD $4^{+}$ 
cell response to Th1 in BALB/c mice, and that both cytokines synergize with IL-12 in inducing cytotoxic T-lymphocytes. ${ }^{29}$ It is possible that the triple combination of IL-12, TNF- $\alpha$, and GM-CSF may be the most potent combination, ${ }^{25}$ but this was not investigated in this series of experiments. The combination of TNF- $\alpha$ and GM-CSF was also studied but did not demonstrate any significant benefit (data not shown).

These results suggest that a single intratumoral injection of IL-12 and TNF- $\alpha$-loaded PLAM into a primary breast cancer prior to surgical excision can lead to a tumor-specific immune response. We have previously demonstrated that this systemic antitumor response stimulated in the neoadjuvant setting is capable of eradicating micrometastatic disease, thus reducing both local and distant recurrence. ${ }^{7}$ There is evidence that immunotherapy may be most successful in the minimal residualdisease setting ${ }^{30,31}$ and is less likely to be effective in the setting of advanced disease. ${ }^{14,32,33}$ Therefore, stimulating an immune response in the neoadjuvant or adjuvant period appears to be the most likely approach for clinical impact.

In conclusion, the local and sustained release of IL-12 and TNF- $\alpha$ within a breast tumor, as can be accomplished by a single intratumoral injection of the cytokines encapsulated in poly-lactic-acid microspheres, can lead to regression of the primary tumor and, more important, the generation of a tumor-specific T-cell-mediated immune response. This approach holds several advantages regarding clinical feasibility in comparison with many immunotherapeutic approaches being investigated in breast cancer. Several questions remain regarding the clinical application of cytokine-encapsulated microspheres, including the potential impact of surgery, radiation therapy, or systemic therapies on the immune response. Further investigation into the mechanisms involved in both the local and distant effects are warranted so that this approach can be translated to the clinic.

\section{ACKNOWLEDGMENTS}

The acknowledgments are available online in the fulltext version at www.annalssurgicaloncology.org. They are not available in the PDF version.

\section{REFERENCES}

1. Riethmuller G, Johnson JP. Monoclonal antibodies in the detection and therapy of micrometastatic epithelial cancer. Curr Opin Immunol 1992;4:647-55.

2. Brown DC, Purushotham AD, Birnie GD, George WD. Detection of intraoperative tumor cell dissemination in patients with breast cancer by use of reverse transcription and polymerase chain reaction. Surgery 1995;117:96-101.
3. Braun S, Kentenich C, Janni W, et al. Lack of effect of adjuvant chemotherapy on elimination of single dormant tumor cells in bone marrow of high risk breast cancer patients. J Clin Oncol 2000;18: $80-6$.

4. Ghadersohi A, Chitta K, Greco WR, et al. Tumor antigens and markers for breast and ovarian cancers. Frontiers in Bioscience 2002; 7:e48-57.

5. Mastrangelo MJ, Sato T, Lattime EC, et al. Cellular vaccine therapies for cancer. Cancer Treat Res 1998;94:35-50.

6. Egilmez NK, Jong YS, Sabel MS, et al. In situ tumor vaccination with interleukin-12 encapsulated biodegradable microspheres: induction of tumor regression and potent antitumor immunity. Cancer Res 2000;60:3832-7.

7. Sabel MS, Hill HC, Jong YS, et al. Neoadjuvant therapy with interleukin-12 loaded polylactic acid microspheres reduces local recurrence and distant metastases. Surgery 2001;130:470-8.

8. Margaretten NC, Witschi H. Effects of hyperoxia on growth characteristics of metastatic murine tumors in the lung. Cancer Res 1988;48:2779-83.

9. Aruga E, Aruga A, Arca MJ, et al. Immune responsiveness to a murine mammary carcinoma modified to express B7-1, IL-12 or GM-CSF. Cancer Gene Therapy 1997;4:157-66.

10. Wiltrout RH, Matheison BJ, Back TC, Salup RR. Therapeutic requirements for the successful treatment of murine renal carcinoma by adoptive chemoimmunotherapy. Progr Clin Biol Res 1987;244:17-25.

11. Yuhas JM, Pazmino NH, Proctor JO, Toya RE. A direct relationship between immune competence and the subcutaneous growth rate of a malignant murine lung tumor. Cancer Res 1974;34: $722-8$.

12. Mathiowitz E, Jacob JS, Jong YS, et al. Biologically erodable microspheres as potential oral drug delivery systems. Nature 1997; 386:410-4.

13. Yoshizawa H, Sakai K, Chang AE, Shu S. Activation of anti-CD3 of tumor draining lymph node cells for specific adoptive immunotherapy. Cell Immunol 1991;134:473-9.

14. Parmiani G, Rodolfo M, Melani C. Immunological gene therapy with ex vivo gene-modified tumor cells: a critique and an reappraisal. Hum Gene Ther 2000;11:1269-75.

15. Hill HC, Conway TF Jr, Sabel MS, et al. Cancer Immunotherapy with interleukin-12 and granulocyte-macrophage colony-stimulating factor-encapsulated microspheres: coinduction of innate and adaptive antitumor immunity and cure of disseminated disease. Cancer Res 2003;62:7254-63.

16. Burke F. Cytokines (IFNs, TNF-alpha, IL-2, and IL-12) and animal models of cancer. Cytokines Cell Mol Ther 1999;5:51-61.

17. Hendrzak JA, Brunda MJ. Antitumor and antimetastatic activity of interleukin 12. Curr Top Microbiol Immunol 1996;213:65-83.

18. Brunda MJ, Luistro L, Warrier RR, et al. Antitumor and antimetastatic activity of interleukin 12 against murine tumors. $J$ Exper Med 1993;178:1223-30.

19. Brunda MJ, Luistro L, Rumennik L, et al. Interluekin-12: murine models of a potent antitumor agent. Ann NY Acad Sci 1996;795: $266-74$.

20. Reference deleted.

21. Smyth MJ, Taniguchi M, Street SEA. The anti-tumor activity of IL-12: mechanisms of innate immunity that are model and dose dependent. J Immunology 2000;165:2665-70.

22. Gee MS, Koch CJ, Evans SM, et al. Hypoxia-mediated apoptosis from angiogenesis inhibition underlies tumor control by recombinant interleukin 12. Cancer Res 1999;59:4882-9.

23. Trinchieri G. Interleukin-12: a cytokine at the interface of inflammation and immunity. Adv Immunol 1998;70:83-243.

24. Aruga A, Tanigawa K, Aruga E, et al. Enhanced adjuvant effect of granulocyte-macrophage colony stimulating factor plus interleukin-12 compared with either alone in vaccine-induced tumor immunity. Cancer Gene Therapy 1999;6:89-95.

25. Ahlers JD, Belyakov IM, Matsui S, Berzofsky JA. Mechanisms of 
cytokine synergy essential for vaccine protection against viral challenge. Intern Immunol 2001;13:897-908.

26. Lasek W, Feleszko W, Golab J, et al. Antitumor effects of the combination immunotherapy with interleukin-12 and tumor necrosis factor alpha in mice. Cancer Immunol Immunother 1997;45: $100-8$.

27. Lasek W, Mackiewicz A, Czajka A, et al. Antitumor effects of the combination therapy with TNF-alpha gene-modified tumor cells and interleukin 12 in a melanoma model in mice. Cancer Gene Ther 2000;7:1581-90.

28. Zagozdzon R, Stoklosa T, Golab J, et al. Augmented antitumor effects of combination therapy with interleukin-12, cisplatin, and tumor necrosis factor-alpha in a murine melanoma model. Anticancer Res 1997;17:4493-8.

29. Ahlers JD, Dunlop N, Alling DW, et al. Cytokine-in-adjuvant steering of the immune response phenotype to HIV-1 vaccine constructs: GM-CSF and TNF-a synergize with IL-12 to enhance induction of CTL. J Immunol 1997;158:3947-58.

30. Simon RM, Steinberg SM, Hamilton M, et al. Clinical trial designs for the early clinical development of therapeutic cancer vaccines. J Clin Oncol 2001;19:1848-54.

31. Bendandi M, Gocke CD, Kobrin CB, et al. Complete molecular remissions induced by patient-specific vaccination plus granulocyte-monocyte colony stimulating factor against lymphoma. Nat Med 1999;5:1171-7.

32. Kiessling R, Wasserman K, Horiguchi S, et al. Tumor-induced immune dysfunction. Cancer Immunol Immunother 1999;48:353-62.

33. Hsieh CL, Chen DS, Hwang LH. Tumor-induced immunosuppression: a barrier to immunotherapy of large tumors by cytokinesecreting tumor vaccine. Hum Gene Ther 2000;11:681-92. 